\title{
Comparison of short-term outcomes following pelvic reconstruction with Perigee and Apogee systems: hysterectomy or not?
}

\author{
Li-Ching Chu $\cdot$ Fei-Chi Chuang $\cdot$ Fu-Tsai Kung • \\ Kuan-Hui Huang
}

Received: 5 March 2011 / Accepted: 11 July 2011 /Published online: 6 August 2011

(C) The Author(s) 2011. This article is published with open access at Springerlink.com

\begin{abstract}
Introduction and hypothesis This study aims to compare the surgical outcomes between hysterectomy and uterine preservation in pelvic reconstruction with Perigee ${ }^{\circledR}$ and Apogee ${ }^{\circledR}$ systems for severe pelvic organ prolapse.

Methods Ninety-one women who have undergone transvaginal pelvic reconstructive surgery with Perigee and Apogee systems for severe pelvic organ prolapse were divided into two groups: hysterectomy $(n=39)$ and uterine preservation $(n=52)$. The pre-operative and post-operative assessments include subjective urinary and prolapse symptoms, objective pelvic organ prolapse quantification (POP-Q) system, urodynamic examination, and complications.

Results The mean follow-up period was 8.9 months (range, 0.9-26.5). There were no anatomical differences between the two groups other than a longer perineal body in the hysterectomy group (3.9 vs. 3.6, $p<0.05)$ and a longer total vaginal length in the uterine preservation group (8.2 vs. 7.8, $p<0.05$ ). Preservation of uterus has significantly reduced operative time, blood loss, and days of urine indwelling catheter $(p<0.001)$.

Conclusions Hysterectomy and uterine preservation have comparable anatomical outcomes and post-operative complications in pelvic reconstruction with Perigee and Apogee systems at short-term follow-up.
\end{abstract}

Keywords Pelvic organ prolapse $\cdot$ Perigee $\cdot$ Apogee . Hysterectomy $\cdot$ Uterine preservation

L.-C. Chu $\cdot$ F.-C. Chuang $\cdot$ F.-T. Kung $\cdot$ K.-H. Huang $(\bowtie)$

Department of Obstetrics and Gynecology,

Kaohsiung Chang Gung Memorial Hospital and Chang Gung

University College of Medicine,

No.123, Dapi Rd Niaosong Dist. 833 Kaohsiung, Taiwan

e-mail: gynh2436@adm.cgmh.org.tw

\author{
Abbreviations \\ POP Pelvic organ prolapse \\ POP-Q POP quantification \\ TOT Transobturator tape
}

\section{Introduction}

It has been estimated that approximately $50 \%$ of parous women suffer from pelvic organ prolapse (POP) and $11 \%$ of all women up to the age of 80 years will have surgery for POP or urinary incontinence, with a reoperation rate of $29 \%[1,2]$. The success observed with the use of mesh in general surgery, combined with the high failure rates for traditional colporrhaphy, have led to new surgical approaches and reconstructive materials in gynecologic reconstructive surgery. In 2004, the Food and Drug Administration approved the use of commercial kits that delivered polypropylene mesh through a transvaginal technique. Commercial graft kits have been developed in response to high prolapse recurrence rates and desire for minimally invasive, time-efficient procedures. Perigee/ Apogee $^{\circledR}$ (American Medical Systems, Minnetoka, MN, USA) is one of such kits applied for the pelvic reconstruction of the anterior, middle, and posterior compartments defects. This can be achieved in patients without a uterus, or the mesh can be used to re-suspend a retained uterus.

For correction of POP, hysterectomy is considered as a standard practice even though descent of the uterus is a consequence and not the cause of prolapse [3]. Will hysterectomy help or hinder our efforts to achieve a successful and long lasting anatomical result for woman with POP? The answer to this question is still under debate and needs to be considered from both patient and physician 
perspectives. Few studies on uterine preservation have been reported, and there are no clear indications for uterus sparing or removal in open or vaginal surgery for advanced prolapse [4-6]. Because uterine preservation or not with both Perigee and Apogee have not been directly compared in the literature, the appropriate management of a patient scheduled for Perigee and Apogee with a uterus in situ remains unclear. In this study, we performed both Perigee and Apogee in women with advanced POP and compared the peri- and post-operative outcomes between groups of uterine preservation and hysterectomy.

\section{Materials and methods}

From May 2008 to September 2010, 123 consecutive patients with symptomatic POP quantification (POP-Q) system stage III-IV, who underwent transvaginal pelvic reconstructive surgery with Perigee and Apogee systems at our hospital were recruited [7]. Three patients were excluded because of missing POP-Q records and another 29 patients were excluded as well for previous hysterectomy history with vault prolapse. The remaining 91 women with uterus in situ were divided into the hysterectomy group $(n=39)$ and the uterine preservation group $(n=52)$.

Patients had been interviewed to identify urinary and prolapse symptoms. The POP-Q standard scoring system and the $3 \times 3$ grid system were adopted for staging and recording the nine points of the POP-Q system. Preoperative POP-Q values and stages were measured under anesthesia in operative room while post-operative POP-Q values and stages were measured at each clinical follow-up. All POP-Q points were measured by the corresponding author (K.-H. Huang). Baseline demographic data including age, body mass index, obstetric and gynecologic history, and medical and surgical history were obtained. The following pre-operative and peri-operative information were also collected: urinalysis taken $18-24 \mathrm{~h}$ prior to surgery, hemoglobin taken $18-24 \mathrm{~h}$ before and after surgery, operative time (from skin incision to skin closure), estimated blood loss, days of urine indwelling catheter, length of stay in hospital recorded in days, and perioperative complications. All the women without postmenopausal bleeding, previous cervical intraepithelial neoplasia (CIN), abnormal cervical smears, or uterine diseases were offered the option to preserve the uterus.

All patients have undergone pre-operative multichannel urodynamic studies with prolapse reduction to determine whether there was any evidence of urodynamic stress incontinence or occult stress urinary incontinence. Those with confirmed urodynamic stress incontinence had placement of a transvaginal midurethral polypropylene sling (Transobturator tape $\left(\mathrm{TOT}^{\circledR}\right)$, American Medical Systems, Minnetoka, MN,
USA) at the same time. Cystoscopy was performed on all patients after mesh was placed intraoperatively to exclude any bladder injury and to confirm intact ureters.

All cases were performed by a single surgeon (Dr. K.-H. H.) using the technique outlined by the manufacturer with only subtle differences in the Apogee procedure involved [8]. Dr. K.-H. H. passed the needle through the sacrospinous ligament at a level of $2 \mathrm{~cm}$ posterior and medial to the ischial spine rather than the ileococcygeus muscle at the level of the ischial spine. All patients were given antibiotic prophylaxis (intravenous Cefazolin $1 \mathrm{gm}$; Chi-Sheng Chemical Co., Hsinchu, Taiwan) administered $30 \mathrm{~min}$ before surgery and till 2 to 3 days (intravenous Cefazolin $1 \mathrm{gm}$ every $8 \mathrm{~h}$ ) after surgery. General anesthesia was applied in all operations.

Post-operative follow-up was scheduled at $1,3,6$, and 12 months and then annually. Primary post-operative data consisted of POP-Q values, stage of prolapse, and complications. Urinary symptoms of complication included dysuria, urine retention, confirmed urinary tract infection, nocturia, frequency, urgency, urgent incontinence, and stress incontinence using the 2002 ICS definitions [9]. Abnormal sensation was defined as groin, vaginal, buttock, or thigh pain; numbness; or weakness. Mesh extrusion was confirmed by pelvic examination. Symptoms or signs which did not fit descriptions above were classified under the category of other complication. Recurrence was defined as most distal portion of POP stage II or greater.

Charts were reviewed by computerized hospital records. Data were collected and reviewed by the first author. Ethical approval by the Institutional Review Board of our hospital had been obtained for retrospective data analysis. Continuous variables were summarized with means and standard deviations and were compared between groups using Student's $t$ test. Categorical variables were summarized with counts and percents and were compared between groups using chisquare test with Fisher's exact test, if necessary. The change from pre- to post-operative status for POP-Q values was assessed using paired Student's $t$ tests. A difference was considered statistically significant when $p<0.05$.

\section{Results}

Comparison of baseline characteristic between the two groups: hysterectomy and uterine preservation are listed in Table 1. No significant differences between the two groups were identified, except for the uterine preservation group had older age ( 65.5 vs $59.3 ; p=0.003$ ), higher parity ( 3.9 vs 3.3 ; $p=0.02)$, and more patients with menopause $(98.1 \%$ vs $66.7 \% ; p<0.001)$. The mean follow-up period was 8.9 months (range, 0.9-26.5). There was no significant difference of the follow-up time between the two groups $(p=0.249)$. 
Table 1 Baseline characteristics in both groups

\begin{tabular}{llll}
\hline Variable & Hysterectomy $(n=39)$ & Uterine preservation $(n=52)$ & $P$ value \\
\hline Age (years) & $59.3 \pm 10.7$ & $65.5 \pm 8.0$ & $0.003^{\mathrm{a} *}$ \\
Parity & $3.3 \pm 1.4$ & $3.9 \pm 1.1$ & $0.020^{\mathrm{a} *}$ \\
BMI $\left(\mathrm{kg} / \mathrm{m}^{2}\right)$ & $25.3 \pm 2.4$ & $24.9 \pm 3.4$ & $0.600^{\mathrm{a}}$ \\
Menopause & $26(66.7)$ & $51(98.1)$ & $<0.001^{\mathrm{b} *}$ \\
Diabetes Mellitus & $11(28.2)$ & $9(17.3)$ & $0.306^{\mathrm{b}}$ \\
Hypertension & $14(35.9)$ & $25(48.1)$ & $0.288^{\mathrm{b}}$ \\
History of POP repair & $1(2.6)$ & $2(3.8)$ & $35(67.3)$ \\
Concomitant transobturator tape (TOT) & $24(61.5)$ & $12.9 \pm 1.2$ & $0.99^{\mathrm{b}}$ \\
Pre-operative hemoglobin (gm/dL) & $13.0 \pm 1.4$ & $15(28.8)$ & $0.659^{\mathrm{b}}$ \\
Pre-operative urinalysis (positive) & $10(25.6)$ & $9.4 \pm 7.2$ & $0.737^{\mathrm{a}}$ \\
Follow-up time (months) & $7.9 \pm 5.3$ & & $0.815^{\mathrm{b}}$ \\
\hline
\end{tabular}

Data are given as mean \pm standard deviation, or $n(\%)$

$B M I$ body mass index, $P O P$ pelvic organ prolapse

${ }^{\text {a }}$ Student's $t$ test

${ }^{\mathrm{b}}$ Chi-square test with Fisher's exact test

$* p<0.05$, statistically significant

Primary post-operative outcome analyses comparing the pre-operative and post-operative POP-Q values and the stage of prolapse between the hysterectomy and uterine preservation groups are summarized in Tables 2 and 3, respectively. All the parameters of POP-Q values (Aa, Ba, C, $\mathrm{Ap}, \mathrm{Bp}$, genital hiatus, perineal body, and total vaginal length) and stages have significant improvements $(p<0.001)$ in both groups after surgery except for total vaginal length in the hysterectomy group ( $p=0.815$; Table 2 ). Post-operative POP$\mathrm{Q}$ values and stages between the two groups did not differ significantly ( $p>0.05$; Tables 2 and 3$)$ except for perineal body (hysterectomy, 3.9; uterine preservation, 3.6; $p=0.032$ ) and total vaginal length (hysterectomy, 7.8; uterine preservation, $8.2 ; p=0.04$; Table 2).

Peri-operative outcomes were compared between the two surgical groups and are summarized in Table 4. Operative time (129.3 vs 97.2; $p<0.001)$, estimated blood loss (179.5 vs $77.4 ; p<0.001)$, and days of urine indwelling catheter ( 2.7 vs $2.4 ; p=0.006$ ) of the hysterectomy group were significantly higher than those in the uterine preservation group. There was no significant difference between the two groups in post-operative hemoglobin (11.1 vs $11.4, p=0.370$ ), hospital stay (5.3 vs $4.9 ; p=0.103$ ), and complications, including urinary symptoms, abnormal sensation, or symp-

Table 2 Pre-operative and post-operative pelvic organ prolapse quantification (POP-Q) values in both groups

\begin{tabular}{|c|c|c|c|c|c|c|c|}
\hline \multirow[t]{2}{*}{ POP-Q variables $(\mathrm{cm})$} & \multicolumn{3}{|c|}{ Hysterectomy $(n=39)$} & \multicolumn{3}{|c|}{ Uterine preservation $(n=52)$} & \multirow[t]{2}{*}{ Post-op $p$ value } \\
\hline & Pre-op & Post-op & $p$ Value $^{\mathrm{a}}$ & Pre-op & Post-op & $p$ Value $^{\mathrm{a}}$ & \\
\hline $\mathrm{Aa}$ & $+2.9 \pm 0.3$ & $-3.0 \pm 0.0$ & $<0.001^{*}$ & $+2.9 \pm 0.3$ & $-3.0 \pm 0.2$ & $<0.001^{*}$ & 0.159 \\
\hline $\mathrm{Ba}$ & $+4.8 \pm 1.2$ & $-3.2 \pm 0.9$ & $<0.001 *$ & $+4.6 \pm 1.1$ & $-3.1 \pm 0.6$ & $<0.001 *$ & 0.352 \\
\hline $\mathrm{C}$ & $+4.4 \pm 1.8$ & $-7.6 \pm 0.9$ & $<0.001 *$ & $+4.0 \pm 1.7$ & $-7.7 \pm 0.9$ & $<0.001 *$ & 0.582 \\
\hline Ap & $+2.7 \pm 0.7$ & $-3.0 \pm 0.0$ & $<0.001 *$ & $+2.4 \pm 0.7$ & $-3.0 \pm 0.0$ & $<0.001 *$ & $=0.99$ \\
\hline $\mathrm{Bp}$ & $+3.7 \pm 1.5$ & $-3.1 \pm 0.8$ & $<0.001 *$ & $+3.0 \pm 1.4$ & $-3.1 \pm 0.7$ & $<0.001 *$ & 0.839 \\
\hline Gh & $4.8 \pm 0.9$ & $3.7 \pm 0.9$ & $<0.001 *$ & $5.0 \pm 0.7$ & $3.6 \pm 0.8$ & $<0.001 *$ & 0.666 \\
\hline $\mathrm{Pb}$ & $3.2 \pm 0.6$ & $3.9 \pm 0.4$ & $<0.001 *$ & $3.0 \pm 0.6$ & $3.6 \pm 0.7$ & $<0.001 *$ & $0.032 *$ \\
\hline tvl & $7.7 \pm 1.2$ & $7.8 \pm 1.0$ & 0.815 & $7.5 \pm 0.9$ & $8.2 \pm 0.8$ & $<0.001^{*}$ & $0.040^{*}$ \\
\hline
\end{tabular}

Data are given as mean \pm standard deviation

Pre-op pre-operative, Post-op post-operative, $g h$ genital hiatus, $p b$ perineal body, $t v l$ total vaginal length

${ }^{\text {a }}$ Student's $t$ test

${ }^{*} p<0.05$, statistically significant 
Table 3 Pre-operative and post-operative pelvic organ prolapse quantification (POP-Q) stages in both groups

\begin{tabular}{|c|c|c|c|c|c|c|c|}
\hline \multirow{2}{*}{$\begin{array}{l}\text { POP-Q stage } \\
\text { Vaginal wall }\end{array}$} & \multicolumn{3}{|c|}{ Hysterectomy $(n=39)$} & \multicolumn{3}{|c|}{ Uterine preservation $(n=52)$} & \multirow[t]{2}{*}{ Post-op $p$ value } \\
\hline & Pre-op & Post-op & $p$ Value $^{\mathrm{a}}$ & Pre-op & Post-op & $p$ Value $^{\mathrm{a}}$ & \\
\hline Anterior & $3.9 \pm 0.3$ & $0.9 \pm 0.2$ & $<0.001 *$ & $3.9 \pm 0.3$ & $1.0 \pm 0.1$ & $<0.001 *$ & 0.402 \\
\hline Apex & $3.7 \pm 0.7$ & $0.0 \pm 0.0$ & $<0.001^{*}$ & $3.6 \pm 0.7$ & $0.0 \pm 0.0$ & $<0.001^{*}$ & $=0.99$ \\
\hline Posterior & $3.5 \pm 0.6$ & $1.0 \pm 0.2$ & $<0.001 *$ & $3.2 \pm 0.6$ & $1.0 \pm 0.1$ & $<0.001 *$ & 0.839 \\
\hline
\end{tabular}

Data are given as mean \pm standard deviation

${ }^{a}$ Student's $t$ test

${ }^{*} p<0.05$, statistically significant

toms and signs that did not fit into the categories of urinary symptoms or abnormal sensation $(p>0.05)$.

Secondary outcome analyses showed that there was no significant differences between the two groups for mesh extrusion (hysterectomy, 12.8\%; uterine preservation, 3.8\%; $p=0.134$ ), urinary symptoms (hysterectomy, $30.8 \%$; uterine preservation, 44.2\%; $p=0.276$ ), abnormal sensation (hysterectomy, 7.7\%; uterine preservation, $5.8 \%$; $p=0.99$ ), and other post-operative complications (hysterectomy, 17.9\%, uterine preservation, $7.7 \% ; p=0.195$; Table 5). There was no major peri-operative complication except for one blood transfusion with two units of packed RBC in the hysterectomy group, and no rectum or bladder perforation during operation in both groups.

There was an approximately 98\% anatomical cure rate (hysterectomy, 39 out of 39, 100\%; uterine preservation, 50 out of 52, 96\%; at POP-Q stage I or less) of severe pelvic organ prolapse. Two recurrent cystocele POP-Q stage II was both noted in uterine preservation group 7 and 10 months after surgery, respectively.

\section{Discussion}

Concomitant hysterectomy or uterine preservation of vaginal mesh surgeries for pelvic organ prolapse are still under debate and have not been adequately addressed in the literature. There are several case series describing results after use of vaginal mesh; however, most of these studies combine the outcomes of Perigee and/or Apogee, Prolift ${ }^{\circledR}$ (Women's Health and Urology/Ethicon, Somerville, NJ, USA) insertions and do not stratify according to hysterectomy versus uterine preservation $[10,11]$. There was a study investigated the outcomes related to total Prolift with and without leaving the uterus in situ [12]. But the comparison of outcomes related to Perigee and Apogee with and without hysterectomy has not been adequately addressed. This study attempted to assess the differences between using Perigee and Apogee with and without hysterectomy.

All the women without post-menopausal bleeding, previous CIN, abnormal cervical smears, or uterine disease

Table 4 Peri-operative outcomes

\begin{tabular}{llll}
\hline Variable & Hysterectomy $(n=39)$ & Uterine preservation $(n=52)$ & $p$ Value \\
\hline Operative time (min) & $129.3 \pm 27.5$ & $97.2 \pm 19.2$ & $<0.001^{\mathrm{a} *}$ \\
Estimated blood loss (ml) & $179.5 \pm 127.1$ & $77.4 \pm 42.9$ & $<0.001^{\mathrm{a} *}$ \\
Post-operative hemoglobin (gm/dL) & $11.1 \pm 1.4$ & $11.4 \pm 1.0$ & $0.370^{\mathrm{a}}$ \\
Duration of urine indwelling catheter (days) & $2.7 \pm 0.5$ & $2.4 \pm 0.5$ & $0.006^{\mathrm{a} *}$ \\
Hospital stay (days) & $5.3 \pm 1.0$ & $4.9 \pm 1.1$ & $0.103^{\mathrm{a}}$ \\
Complications & & & \\
None & $35(89.7)$ & $43(82.7)$ & $7(13.5)$ \\
Urinary symptoms & $2(5.1)$ & $1(1.9)$ & $0.383^{\mathrm{b}}$ \\
Abnormal sensation & $1(2.6)$ & $1(1.9)$ & $0.291^{\mathrm{b}}$ \\
Other & $1(2.6)$ & $=0.99^{\mathrm{b}}$ \\
\hline
\end{tabular}

Data are given as mean \pm standard deviation, or $n(\%)$

${ }^{\text {a }}$ Student's $t$ test

${ }^{\mathrm{b}}$ Chi-square test with Fisher's exact test

${ }^{*} p<0.05$, statistically significant 
Table 5 Post-operative complications

\begin{tabular}{lccc}
\hline Variable & Hysterectomy $(n=39)$ & Uterine preservation $(n=52)$ & $p$ Value $^{\mathrm{a}}$ \\
\hline None & $12(30.8)$ & $20(38.5)$ & 0.510 \\
Mesh extrusion & $5(12.8)$ & $2(3.8)$ & 0.134 \\
Urinary symptoms & $12(30.8)$ & $23(44.2)$ & 0.276 \\
Abnormal sensation & $3(7.7)$ & $3(5.8)$ & $=0.99$ \\
Other & $7(17.9)$ & $4(7.7)$ & 0.195 \\
\hline
\end{tabular}

were offered the chance to preserve the uterus. In this study, the hysterectomy group had significantly younger age, less parity, and fewer patients with menopause. By reviewing the charts, we found that more patients with uterine myomas or menstrual discomforts such as dysmenorrhea and menorrhagia were noted in the hysterectomy group (23 out of 39). Most of these symptomatic patients who received hysterectomy were in reproductive age. This could explain the causes of younger age, less parity, and less patients with menopause in the hysterectomy group.

Our study also showed that POP-Q values and the stage of prolapse significantly improved after both surgeries, and there were no significant differences in post-operative POP$\mathrm{Q}$ values and stages in any vaginal compartment of both groups. This might suggest that either hysterectomy or uterine preservation group has similar anatomical outcomes in pelvic reconstruction for severe pelvic organ prolapse at short-term follow-up. However, less elongation of total vaginal length after surgery was noted in the hysterectomy group. That is probably because of partial vaginal wall was cut along with the uterine cervix during procedure of hysterectomy. Longitudinal suturing of vaginal cuff after removal of uterus might increase the vaginal length.

There was no significant difference between the two groups in peri-operative outcomes and post-operative complications except that the uterine preservation group has significantly reduced operative time, estimated blood loss, and days of urine indwelling catheter. This indicated that both hysterectomy and uterine preservation groups are equally safe but the uterine preservation group had the advantage of shorter operative time, reduced estimated blood loss, and fewer days of urine indwelling catheter. Hysterectomy may result in damage to the nerve supply or supportive tissues of the pelvic floor, which may lead to subsequent pelvic floor dysfunction and cause longer days of urine indwelling catheter post-operatively. Though the days of an indwelling catheter of 2.7 vs. 2.4 days seem not to be clinically important, there is still 0.3 day $=7.2 \mathrm{~h}$ difference between the two groups. We think patients may have more discomforts with a longer duration of $7.2 \mathrm{~h}$ of urine indwelling catheter. But this is a priori without subjective questionnaires. If the longer duration of urine indwelling catheter increased the infection rate also remains unclear without post-operative urinalysis or urine culture.
We would collect more subjective and objective data to clarify the impact of duration of urine indwelling catheter on clinical condition in future studies. Furthermore, the average hospital stay was about 5 days in our study for a vaginal surgery. This is far more than the average stay in most western countries (less than 1 day in the USA and Canada). The discrepancy is probably because of the convenient and affordable medical treatment in Taiwan and cultural difference. The rate of doctor visits and hospitalizations is higher than in most western countries since the Taiwan's national health insurance started in 1995 (information on the Web site of Bureau of National Health Insurance http://www.nhi.gov.tw/english/index.aspx). Each patient in our study only had to pay average 300 USD for her hospitalization and surgery (not including the materials expenses of Perigee and Apogee about 2,000 USD).

Recently, concern over complications with vaginal mesh has been raised. Complications that may be related to the mesh itself, such as mesh vaginal extrusion, pain (vaginal, groin, buttock, or leg), dyspareunia, infection, or fistula, have been reported in the literature $[13,14]$. The rate of mesh extrusion in this study is consistent with other published reports of type I mesh complications at $3 \%$ to $12 \%[15,16]$. In the present study, the rate of mesh extrusion was higher in the hysterectomy group (hysterectomy, $12.8 \%$; uterine preservation, $3.8 \%$ ) but was not statistically significant $(p=0.134$; Table 5). Collinet et al. proposed that uterine preservation is a protective factor of mesh extrusion and reported an increased relative risk of 5.16 compared with the situation when the uterus was preserved or when the patient had had a hysterectomy in the past [14]. The clinically significant mesh extrusion difference in our study correlated with this observation. Besides, more complications seemed to have been noted at clinical follow-up, but follow-up beyond 12 months could therefore reveal more significant differences between the two groups and between the peri-operative and post-operative periods for complications.

Should we expect uterine preservation to be better than hysterectomy in pelvic reconstruction for pelvic organ prolapse - and could it become the surgical gold standard in the future? It might be too early to draw any definite conclusions. In this study, we have brought up an alternative procedure in pelvic reconstruction for severe pelvic organ prolapse: the uterine preservation which is potentially 
effective and minimally invasive. We offered choices and let patients know that hysterectomy was not the only option.

A weakness of the study was that we did not collect data regarding the subjective outcomes of patients and quality of life using standardized questionnaire after the procedure. Postoperative multichannel urodynamic studies were not obtained completely to compare the change from preoperative to postoperative conditions. Other limitations of our study are shorter follow-up of anatomical and functional results, and nonprospective randomized trials. A further study that focuses on the prospective collection of data controlling for baseline characteristics at the time of enrolment, randomizing patients to either hysterectomy or uterine preservation and having follow-up beyond 12 months or longer should be done.

In conclusion, there were no significant differences between the hysterectomy group and the uterine preservation group for post-operative POP-Q values and stages and post-operative complications in pelvic reconstruction with Perigee and Apogee systems for severe pelvic organ prolapse after an average of 8.9 months of follow-up (range, 0.9-26.5). The notable differences between the two procedures were a longer perineal body after hysterectomy and a longer total vaginal length after uterine preservation. Preservation of uterus has the significant advantage of shorter operative time, less blood loss, and fewer days of urine indwelling catheter. Longer follow-up of anatomical and functional results are needed.

Acknowledgments Thanks to Dr. Hung-Chung Fu for his assistance with the statistical analysis.

\section{Conflicts of interest None.}

Open Access This article is distributed under the terms of the Creative Commons Attribution Noncommercial License which permits any noncommercial use, distribution, and reproduction in any medium, provided the original author(s) and source are credited.

\section{References}

1. Beck RP, McCormick S, Nordstrom L (1991) A 25-year experience with 519 anterior colporrhaphy procedures. Obstet Gynecol 78:1011-1018
2. Olsen AL, Smith VJ, Bergstrom JO, Colling JC, Clark AL (1997) Epidemiology of surgically managed pelvic organ prolapse and urinary incontinence. Obstet Gynecol 89:501-506

3. Bonney V (1934) The principles that should underline all operations for prolapse. J Obstet Gynaecol Br Emp 41:669-703

4. Maher CF, Cay MP, Slack MC, Murray CJ, Milligan M, Schluter P (2001) Uterine preservation or hysterectomy at sacrospinous colpopexy for uterovaginal prolapse? Int Urogynecol J 12:384-385

5. van Brummen HJ, van de Pol G, Aalders CIM, Heintz AP, van der Vaart CH (2003) Sacrospinous hysteropexy compared to vaginal hysterectomy as primary surgical treatment for a descensus uteri: effects on urinary symptoms. Int Urogynecol J 14:350-355

6. HefniM El-Toucky T, Bhaumik J, Katsimanis E (2003) Sacrospinous cervicocolpopexy with uterine conservation for uterovaginal prolapse in elderly women: an evolving concept. Am J Obset Gynecol 188:645-650

7. Bump RC, Mattiasson A, Bø K, Brubaker LP, DeLancey JO, Klarskov P et al (1996) The standardization of terminology of female pelvic organ prolapse and pelvic floor dysfunction. Am J Obstet Gynecol 175:10-17

8. Erickson Ty B (2006) Innovations in Surgical Approaches: Perigee $^{\mathrm{TM}}$ and Apogee ${ }^{\mathrm{TM}}$ Placement Kits. OBG Management 18 (suppl):S5-S8

9. Rosier P, Ulmsten U, van Kerrebroeck P, Victor A, Wein A (2002) The standardisation of terminology of lower urinary tract function: report from the Standardisation Sub-committee of the International Continence Society. Neurourol Urodyn 21:167-178

10. Long CY, Long CY, Hsu CS, Jang MY, Liu CM, Chiang PH, Tsai EM (2011) Comparison of clinical outcome and urodynamic findings using "Perigee and/ or Apogee" versus "Prolift anterior and/ or posterior" system devices for the treatment of pelvic organ prolapse. Int Urogynecol J 22:233-239

11. Huang WC, Lin TY, Lau HH, Chen SS, Hsieh CH, Su TH (2011) Outcome of transvaginal pelvic reconstructive surgery with Prolift after a median of 2 years' follow-up. Int Urogynecol J 22:197-203

12. McDermott CD, Terry CL, Woodman PJ, Hale DS (2011) Surgical outcomes following total Prolift: Colpopexy versus hysteropexy. Aust N Z J Obstet Gynaecol 51:61-66

13. Abdel-Fattah M, Ramsay I, West of Scotland Study Group (2008) Retrospective multicentre study of the new minimally invasive mesh repair devices for pelvic organ prolapse. BJOG 115:22-30

14. Marguilies R, Lewicky-Gaupp C, Fenner D, McGuire E, Clemens Q, Delancey J (2008) Complications requiring re-operation following vaginal mesh kit procedures for prolapse. Am J Obstet Gynecol December 678:e1-e4

15. Collinet P, Debodinance P, Ha Duc E, Lucot JP, Cosson M (2006) Transvaginal mesh technique for pelvic organ prolapse repair: mesh exposure management and risk factors. Int Urogynecol $\mathrm{J}$ 17:315-320

16. Fatton B, Amblard J, Debodinance P, Cosson M, Jacquetin B (2007) Transvaginal repair of genital prolapse: preliminary results of new tension-free vaginal mesh (Prolift system)-a case series multicentric study. Int Urogynecol J 18:743-752 